\title{
Microbiology of bile aspirates obtained at ERCP in patients with suspected acute cholangitis
}

\section{다)(우우}

\section{Authors}

Mark A. Gromski ${ }^{*}{ }^{1}$, Aditya Gutta ${ }^{*}, 1$, Glen A. Lehman ${ }^{1}$, Yan Tong ${ }^{2}$, Evan L. Fogel ${ }^{1}$, James L. Watkins ${ }^{1}$, Jeffrey J. Easler ${ }^{1}$, Benjamin L. Bick ${ }^{1}$, Lee McHenry ${ }^{1}$, Cole Beeler ${ }^{3}$, Ryan F. Relich ${ }^{4}$, Bryan H. Schmitt ${ }^{4}$, Stuart Sherman ${ }^{1}$

Institutions

1 Division of Gastroenterology and Hepatology, Department of Medicine, Indiana University School of Medicine, Indianapolis, Indiana, USA

2 Department of Biostatistics, Indiana University School of Medicine, Indianapolis, Indiana, USA

3 Division of Infectious Diseases, Department of Medicine, Indiana University School of Medicine, Indianapolis, Indiana, USA

4 Department of Pathology and Laboratory Medicine, Indiana University School of Medicine, Indianapolis, Indiana, USA

submitted 26.4.2020

accepted after revision 4.3.2022

published online 3.6 .2022

Bibliography

Endoscopy 2022; 54: 1045-1052

DOI 10.1055/a-1790-1314

ISSN 0013-726X

(c) 2022. The Author(s).

This is an open access article published by Thieme under the terms of the Creative Commons Attribution-NonDerivative-NonCommercial License, permitting copying and reproduction so long as the original work is given appropriate credit. Contents may not be used for commercial purposes, or adapted, remixed, transformed or built upon. (https://creativecommons.org/licenses/by-nc-nd/4.0/)

Georg Thieme Verlag KG, Rüdigerstraße 14,

70469 Stuttgart, Germany

Supplementary material

Supplementary material is available under https://doi.org/10.1055/a-1790-1314

Scan this QR-Code for the author commentary.

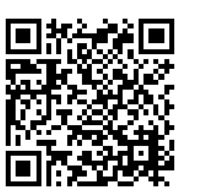

\footnotetext{
* Joint first authors
}

Corresponding author

Mark Andrew Gromski, MD, Department of Medicine, Indiana University School of Medicine, Division of Gastroenterology and Hepatology, 550 N. University Blvd, Suite 1634,

Indianapolis, IN 46202, USA

mgromski@iu.edu

\section{ABSTRACT}

Background The cornerstone of treatment for acute cholangitis is source control with biliary drainage and early antibiotics. The primary aim of this study was to describe the microbiology of bile aspirate pathogens obtained at the time of endoscopic retrograde cholangiopancreatography (ERCP) in patients suspected of having acute cholangitis.

Methods In this single-center retrospective study, patients were included if a bile aspirate was collected at ERCP for suspicion of acute cholangitis, from 1 January 2010 to 31 December 2016.

Results There were 721 ERCP procedures for suspected acute cholangitis with bile culture results, with 662 positive bile cultures (91.8\%). Pathogens included: Enterococcus species (spp.) 448 (67.7\%); Klebsiella spp.295 (44.6\%); Escherichia coli 269 (40.6\%); Pseudomonas spp. 52 (7.9\%); and anaerobes 64 (9.7\%). Susceptibility of Klebsiella pneumoniae and E.coli isolates to ciprofloxacin was $88 \%$ and $64 \%$, respectively. Extended-spectrum beta-lactamases and carbapenem resistance were found in $7.9 \%$ and $3.6 \%$ of Enterobacteriaceae, respectively. There were 437 concurrent blood cultures, of which 174 were positive (39.8\% of cultures drawn). Prior biliary endoscopic sphincterotomy (ES) was evident in 459 ERCP cases (63.7\%), and was associated with increased frequency of Klebsiella spp., Pseudomonas aeruginosa, Enterobacter spp., and Enterococcus spp. Prior biliary ES significantly increased the probability of vancomycin-resistant Enterococcus (VRE).

Conclusions The vast majority of bile cultures (91.8\%) were positive. The susceptibilities of E.coli and K.pneumoniae to ciprofloxacin are lower than historically noted. A notable portion of cultures contained pathogenic drug-resistant organisms. Prior biliary ES is associated with a higher frequency of certain organisms and higher frequency of VRE. 


\section{Introduction}

Acute cholangitis is a frequently encountered clinical entity that can have potentially fatal consequences if not detected and treated promptly. There is a high risk of end-organ damage, leading to a significant mortality rate if treatment is not instituted promptly [1], which drops to $<5 \%$ with timely and appropriate interventions [2]. Treatment for acute cholangitis is incumbent on prompt and adequate hemodynamic resuscitation [3], and initiation of appropriate antimicrobial therapy to limit the local and systemic inflammatory response to sepsis and to contain the spread of infection [3], followed by the achievement of definitive source control by decompressing the biliary tree to relieve biliary obstruction [4]. Biliary decompression is most often achieved by techniques applied at endoscopic retrograde cholangiopancreatography (ERCP). Multiple studies support superior outcomes and improved mortality with ERCP compared with interventional radiology or surgical modalities [5-11]. The selection of appropriate empiric antimicrobial agents in acute cholangitis is an important cornerstone of medical decision-making that is frequently encountered in clinical care.

Our group has previously reported the microbiology of bile cultures aspirated at the time of ERCP in patients with acute cholangitis and cholestasis [12]. In our prior study, the bile aspirates from 160 patients from 1994 to 2000 were analyzed. In the bile aspirates, Escherichia coli, Enterococcus spp., Streptococcus spp., and Klebsiella pneumoniae were most commonly isolated. Polymicrobial infections and Enterococcus infections were more frequently identified in patients with previously placed plastic biliary stents. Based on susceptibility data, ciprofloxacin and ceftriaxone were effective against Gram-negative bacilli in $96 \%$ and $91 \%$ of cases, respectively. This has supported the use of these antibiotics for routine empiric antibiotic coverage in patients with suspected acute cholangitis.

With widespread use of antibiotics and the global emergence of multidrug-resistant organisms, efforts are currently underway to identify microbiological characteristics and recognize patterns of drug resistance related to intra-abdominal infections to help in directing prudent antimicrobial choice, early de-escalation to pathogen-directed therapy, and appropriate termination of therapy. This has prompted prior studies and new recommendations from medical societies reinforcing these principles [2, 13-16].

The primary aim of this study was to describe the microbiology patterns of bile aspirates obtained at the time of ERCP in patients suspected of having acute cholangitis. Secondary aims included: (i) the identification of factors that affect the microbiology patterns, such as the presence/absence of a biliary sphincterotomy, presence/absence of a biliary stent; and (ii) a description of the presence of microbiological resistance among the obtained biliary organisms.

\section{Methods}

This was a single-center retrospective study. The study was approved by the Institutional Review Board (IRB) of Indiana University School of Medicine (study \#1607577831). Patients were included in this study if a bile aspirate was collected at the time of ERCP for clinical suspicion of acute cholangitis, from 1 January 2010 to 31 December 2016. ERCP procedures were carried out at IU Health University Hospital in Indianapolis, Indiana, a large tertiary care teaching hospital. An IRB-approved, prospectively maintained institutional ERCP database and a separate microbiology database were queried to identify patients. The electronic medical record (Cerner, Kansas City, Missouri, USA) and electronic procedure reporting system (ProVation, Minneapolis, Minnesota, USA) were reviewed for the identified patients. Patient demographics, procedure-specific data, and microbial data were collected for each patient and each biliary aspirate.

At our center, bile aspirates are obtained at the time of ERCP at the discretion of the endoscopist, most often in the setting of clinically suspected acute cholangitis. They are routinely drawn for this indication by all endoscopists performing ERCP in our hospital. Very rarely, if the bile is too thick to aspirate, there are concurrent data available that may make bile culture redundant (e. g. a blood culture is already positive for a biliary organism in a patient with pus coming from bile duct), or the endoscopist forgets to obtain the bile specimen, bile may not be aspirated at the time of the ERCP.

For this study, clinical acute cholangitis was defined as the presence of a cholestatic biochemical test profile accompanied by fever, with or without biliary dilatation on imaging, severe abdominal pain, or leukocytosis. Furthermore, patients noted to have purulent bile (milky-white discharge exiting the biliary orifice) at the time of ERCP met the criteria for clinical acute cholangitis. For inpatients undergoing ERCP for suspected acute cholangitis, blood for two sets of aerobic and anaerobic blood culture bottles was routinely drawn within 48 hours of admission (at the clinical discretion of the admitting physician).

Generally, all patients undergoing ERCP for suspected cholangitis have received antibiotics (either as an inpatient, in the emergency room, or pre-procedurally in the ERCP suite). If the patient is an inpatient, their inpatient antibiotic regimen is continued periprocedurally (this is usually intravenous cefepime and metronidazole, or intravenous piperacillin-tazobactam). If the patient is an outpatient, periprocedural antibiotics are given at the discretion of the endoscopist and most often include intravenous cefazolin or ciprofloxacin, depending on the clinical scenario and any medication allergies the patient may have.

The duodenoscope reprocessing techniques during the time period of the study were consistent, with the exception of a more substantial pre-cleaning step and a change to double high level disinfection (DHLD) in May 2015 for all duodenoscopes used at ERCP [17] (Appendix 1s, see online-only Supplementary material). DHLD is one of the four supplementary measures recommended by the US Food and Drug Administration (FDA) for the reprocessing of reusable duodenoscopes $[18,19]$.

Our techniques for collecting, processing, and reporting biliary aspirates during ERCP have been previously described [12]. Our techniques for collecting, processing, and reporting blood cultures during the period of this study are largely unchanged from our previously reported study [12], except for a few mod- 
- Table 1 Characteristics of patients with suspected acute cholangitis undergoing endoscopic retrograde cholangiopancreatography (ERCP) with bile aspiration for culture.

\begin{tabular}{|c|c|c|c|c|}
\hline & Total ERCPs $(n=721)$ & $\begin{array}{l}\text { ERCPs with positive } \\
\text { bile culture }(n=662)\end{array}$ & $\begin{array}{l}\text { ERCPs with negative } \\
\text { bile culture }(n=59)\end{array}$ & $P$ value \\
\hline Age, median (IQR), years & $65(21.0)$ & $65(20.0)$ & $57(34.5)$ & $<0.001$ \\
\hline Sex, female, n (\%) & $290(47.1)$ & $263(39.7)$ & $27(45.8)$ & 0.36 \\
\hline Inpatient, n (\%) & $422(58.5)$ & $403(60.9)$ & $19(32.2)$ & $<0.001$ \\
\hline Choledocholithiasis, n (\%) & $431(59.8)$ & $402(60.7)$ & $29(49.2)$ & 0.41 \\
\hline Malignant stricture, n (\%) & $232(32.2)$ & $226(34.1)$ & $6(10.2)$ & 0.002 \\
\hline Benign stricture, n (\%) & $123(17)$ & $118(17.8)$ & $5(8.5)$ & 0.13 \\
\hline $\begin{array}{l}\text { Primary sclerosing cholangitis, } \\
\mathrm{n}(\%)\end{array}$ & $52(7.2)$ & $49(7.4)$ & $3(5.1)$ & 0.79 \\
\hline $\begin{array}{l}\text { Pus exiting bile duct at time of ERCP, } \\
\mathrm{n}(\%)\end{array}$ & $307(42.5)$ & $296(44.7)$ & $11(18.6)$ & 0.006 \\
\hline
\end{tabular}

ifications. When blood cultures were obtained, they were obtained from patients who were admitted as inpatients. Our techniques for collection and processing of bile cultures and blood cultures are described in Appendix 2s.

\section{Statistical analyses}

Data analysis was performed using STATA13 (StataCorp., LLC, College Station, Texas, USA), SAS9.4 (SAS Inc., Cary, North Carolina, USA), and Excel (Microsoft Corporation, Redmond, Washington, USA). Descriptive data were reported as median and interquartile range (IQR) for continuous variables. Student's $t$-test was used for the comparison of continuous variables. Categorical variables were described using frequency and proportion; the $95 \% \mathrm{Cl}$ of a proportion obtained using the Clopper-Pearson method was also reported. Chi-squared tests or Fisher's exact tests were used for comparisons of categorical data.

A crude odds ratio (OR) and its $95 \% \mathrm{Cl}$ were calculated to compare the relative odds of the occurrence of a particular organism in the bile culture, with relation to prior biliary endoscopic sphincterotomy (ES) and indwelling transpapillary biliary stent. Hochberg's step-up Bonferroni method was used to adjust for multiple tests performed for multiple organisms in the bile culture; the adjusted $P$-values were reported. $P$-values $<0.05$ were considered statistically significant.

\section{Results}

During the study period, a total of 20714 ERCPs were performed at our institution, from which 721 ERCPs with bile aspirates (3.5\%) were identified, from 615 individual patients with suspected acute cholangitis. Some patients had $>1$ ERCP with bile aspiration during the study period, most often in the setting of primary sclerosing cholangitis. The median age of patients at the time of ERCP was 65 years (IQR21).
Of the 721 ERCPs with bile aspirates, the cultures of the bile aspirates were positive in 662 ERCPs (91.8\%), with 403 having been performed on inpatients and 259 on outpatients. Of the negative bile cultures, 19 procedures were performed on inpatients and 40 on outpatients. The characteristics of the patients with suspected acute cholangitis who underwent ERCP with bile aspiration for culture are presented in $>$ Table 1. Patients who had a positive bile culture were significantly older (median 65 vs. 57 years) and were significantly more likely to have purulent bile or pus exiting the bile duct at the time of ERCP, as described by the endoscopist (44.7\% vs. $18.6 \%$ ).

Among the 721 cases in which a bile aspirate was obtained at the time of ERCP, 437 blood cultures were also obtained within 48 hours of presentation, of which 174 (39.8\%) were positive. Of the cases with positive bile cultures who also had blood drawn for cultures, the corresponding blood cultures grew bacterial isolates in $40.6 \%$ of instances (160/394). The microbiological characteristics of the blood cultures obtained are detailed in $>$ Table 2. There was no significant difference in the proportion of patients with positive blood cultures based on whether the corresponding bile culture was positive or negative at the same encounter (40.5\% [95\% Cl35.6\%-45.5\%] vs. 32.6\% [95\% $\mathrm{Cl} 19.1 \%-48.5 \%])$. The most common organism isolated on blood culture was E.coli $(49 / 174 ; 28.2 \%)$.

Of the 662 positive bile cultures, $81.6 \%$ were polymicrobial. The following pathogens were identified (as a percentage of total positive bile cultures aspirated; therefore, owing to polymicrobial cultures, percentages add up to $>100 \%)$ : Enterococcus spp. 448 (67.7\%); Klebsiella spp. 295 (44.6\%); E.coli 269 (40.6 $\%)$; viridans group streptococci 235 (35.5\%); Candida spp. 189 (28.5\%); Pseudomonas spp. 52 (7.9\%); anaerobes 64 (9.7\%; Clostridium spp. 53 and Bacteroides spp. 11); and Staphylococcus aureus 32 (4.8\%). Select antimicrobial susceptibilities of the Gram-negative organisms isolated from bile cultures are presented in Table 3. Furthermore, SPICE organisms (Serratia spp., Pseudomonas spp., Indole-positive Proteus spp., Citrobac- 
- Table 2 Microbiological characteristics of blood cultures for patients with positive or negative bile cultures.

\begin{tabular}{|c|c|c|c|c|c|c|c|}
\hline & \multirow{2}{*}{$\begin{array}{l}\text { Total blood } \\
\text { cultures } \\
(n=437) \\
n\end{array}$} & \multicolumn{2}{|c|}{$\begin{array}{l}\text { Blood cultures with } \\
\text { positive bile culture } \\
(\mathrm{n}=395)\end{array}$} & \multicolumn{2}{|c|}{$\begin{array}{l}\text { Blood cultures with } \\
\text { negative bile culture } \\
(n=43)\end{array}$} & \multirow[t]{2}{*}{$P$ value $^{1}$} & \multirow[t]{2}{*}{$\begin{array}{l}\text { Adjusted } \\
\text { Pvalue }^{2}\end{array}$} \\
\hline & & $\mathbf{n}$ & $\%(95 \% \mathrm{Cl})^{3}$ & $\mathbf{n}$ & $\%(95 \% \mathrm{Cl})^{3}$ & & \\
\hline Positive blood culture & 174 & 160 & $40.5(35.6-45.5)$ & 14 & $32.6(19.1-48.5)$ & 0.31 & $>0.99$ \\
\hline Escherichia coli & 49 & 43 & $10.9(8.0-14.4)$ & 6 & $14.0(5.3-27.9)$ & 0.61 & $>0.99$ \\
\hline Klebsiella spp. & 36 & 36 & $9.1(6.5-12.4)$ & 0 & $0(0-9.2)$ & 0.04 & 0.26 \\
\hline Enterococcus spp. & 15 & 14 & $3.5(2.0-5.9)$ & 1 & $2.3(0.1-12.3)$ & $>0.99$ & $>0.99$ \\
\hline Enterobacter spp. & 10 & 9 & $2.3(1.0-4.3)$ & 1 & $2.3(0.1-12.3)$ & $>0.99$ & $>0.99$ \\
\hline Gram-negative rods, NOS & 25 & 21 & $5.3(3.3-8.0)$ & 4 & $9.3(2.6-22.1)$ & 0.29 & $>0.99$ \\
\hline Other organisms & 39 & 37 & $9.4(6.7-12.7)$ & 2 & $4.7(0.6-15.8)$ & 0.41 & $>0.99$ \\
\hline \multicolumn{8}{|c|}{$\begin{array}{l}\text { spp., species; NOS, not otherwise specified. } \\
1 P \text { value from chi-squared test or Fisher's exact test. } \\
2 \text { Adjusted } P \text { value from Hochberg's step-up Bonferroni method. } \\
{ }^{3} \text { Cls obtained using the Clopper-Pearson method. }\end{array}$} \\
\hline
\end{tabular}

- Table 3 Antimicrobial susceptibilities of isolated Gram-negative bacilli from bile cultures.

\begin{tabular}{|c|c|c|c|c|}
\hline & Ampicillin ${ }^{1}$ & TMP/SMX & Ceftriaxone & Ciprofloxacin \\
\hline Klebsiella pneumoniae & & $183 / 201(91.0 \%)$ & 192/201 (95.5\%) & $176 / 201(87.6 \%)$ \\
\hline Klebsiella oxytoca & & $94 / 94(100 \%)$ & $94 / 94(100 \%)$ & $94 / 94(100 \%)$ \\
\hline Escherichia coli & $145 / 269(53.9 \%)$ & $213 / 269(79.2 \%)$ & $238 / 269(88.5 \%)$ & $173 / 269(64.3 \%)$ \\
\hline Citrobacter freundii complex & & $16 / 17(94.1 \%)$ & $15 / 17(88.2 \%)$ & $17 / 17(100 \%)$ \\
\hline Citrobacter freundii & & $11 / 12(91.7 \%)$ & $9 / 12(75.0 \%)$ & $11 / 12(91.7 \%)$ \\
\hline Other Citrobacter spp. & & $15 / 16(93.8 \%)$ & $15 / 16(93.8 \%)$ & $15 / 16(93.8 \%)$ \\
\hline Pseudomonas aeruginosa & & $0 / 52(0 \%)$ & $0 / 52(0 \%)$ & $24 / 52(46.2 \%)$ \\
\hline Enterobacter cloacae & & $52 / 57(91.2 \%)$ & $49 / 57(86.0 \%)$ & $46 / 57(80.7 \%)$ \\
\hline Enterobacter cloacae complex & & $27 / 28(96.4 \%)$ & $25 / 28(89.3 \%)$ & $27 / 28(96.4 \%)$ \\
\hline Other Enterobacter spp. & & $22 / 23(95.7 \%)$ & $22 / 23(95.7 \%)$ & $21 / 23(91.3 \%)$ \\
\hline Aeromonas spp. & & $11 / 13(84.6 \%)$ & $13 / 13(100 \%)$ & $13 / 13(100 \%)$ \\
\hline Other Gram-negative rods, NOS & & $42 / 48(87.5 \%)$ & $45 / 48(93.8 \%)$ & $44 / 48$ (91.7\%) \\
\hline
\end{tabular}

ter spp., Enterobacter spp., Providentia spp., Morganella spp., and Acinetobacter spp.) with the potential for the inducible $a m p C$ gene (an inducible beta-lactamase-coding gene that is upregulated in response to ceftriaxone, early generation cephalosporins, and extended-spectrum beta-lactam antibiotics) were isolated in 251 bile cultures (37.9\%; identified by organism species, not by $a m p C$ genetic testing). Among Enterobacteriaceae isolates, extended-spectrum beta-lactamase (ESBL)producing organisms were detected in $7.9 \%$ of isolates and carbapenem-resistant Enterobacteriaceae (CRE) in 3.6\%. Of S.aureus isolates $50 \%$ were methicillin-resistant (MRSA), and vanco- mycin resistance (VRE) was detected in $14.7 \%$ of Enterococcus spp. isolates.

Of the 721 total ERCPs, 459 (63.7\%) had a prior biliary ES and $341(47.3 \%)$ had a prior biliary stent. The influence of a prior biliary ES or an indwelling biliary stent on bile cultures are detailed in $>$ Table 4. Bile cultures were significantly more likely to be positive when obtained in the presence of a prior biliary ES (96.1\% [95\%CI93.9\%-97.7\%] vs. 84.4\% [95\%CI79.4\%-88.5 $\%]$; OR4.5). A prior biliary ES significantly increased the probability of VRE in the bile culture $(11.5 \%$ [95\% Cl8.8\%-14.8\%] vs. $5.0 \%$ [95\% Cl2.7\%-8.3\%]; OR2.5]. Certain bacteria were 
- Table4 Associations of prior biliary endoscopic sphincterotomy and indwelling transpapillary biliary stent with bile culture.

\begin{tabular}{|c|c|c|c|c|c|c|c|c|}
\hline & \multicolumn{4}{|c|}{ Biliary endoscopic sphincterotomy } & \multicolumn{4}{|l|}{ Biliary stent } \\
\hline & $\begin{array}{l}\text { Yes } \\
(n=459) \\
n(\%) \\
{[95 \% \mathrm{Cl}]}\end{array}$ & $\begin{array}{l}\text { No } \\
(n=262) \\
n(\%) \\
{[95 \% \mathrm{Cl}]}\end{array}$ & $\begin{array}{l}\text { Crude } \\
\text { odds } \\
\text { ratio } \\
(95 \% \mathrm{Cl})\end{array}$ & $\begin{array}{l}\text { Adjusted } \\
\text { Pvalue }^{1}\end{array}$ & $\begin{array}{l}\text { Yes } \\
\text { (n=341) } \\
n(\%) \\
{[95 \% \mathrm{Cl}]}\end{array}$ & $\begin{array}{l}\text { No } \\
(n=380) \\
n(\%) \\
{[95 \% \mathrm{Cl}]}\end{array}$ & $\begin{array}{l}\text { Crude odds } \\
\text { ratio }(95 \% \mathrm{Cl})\end{array}$ & $\begin{array}{l}\text { Adjusted } \\
\text { value }^{1}\end{array}$ \\
\hline Positive bile culture & $\begin{array}{l}441(96.1) \\
{[93.9-97.7]}\end{array}$ & $\begin{array}{l}221(84.4) \\
{[79.4-88.5]}\end{array}$ & $\begin{array}{l}4.5 \\
(2.6-8.1)\end{array}$ & $<0.001$ & $\begin{array}{l}335(98.2) \\
{[96.2-99.4]}\end{array}$ & $\begin{array}{l}327(86.1) \\
{[82.2-89.4]}\end{array}$ & $\begin{array}{l}9.0 \\
(3.8-21.3)\end{array}$ & $<0.001$ \\
\hline \multicolumn{9}{|c|}{ Multidrug resistant organisms } \\
\hline ESBL & $\begin{array}{l}31(6.8) \\
{[4.6-9.4]}\end{array}$ & $\begin{array}{l}14(5.3) \\
{[3.0-8.8]}\end{array}$ & $\begin{array}{l}1.3 \\
(0.7-2.5)\end{array}$ & 0.93 & $\begin{array}{l}26(7.6) \\
{[5.0-11.0]}\end{array}$ & $\begin{array}{l}19(5.0) \\
{[3.0-7.7]}\end{array}$ & $\begin{array}{l}1.6 \\
(0.9-2.9)\end{array}$ & 0.94 \\
\hline MRSA & $\begin{array}{l}12(2.6) \\
{[1.4-4.5]}\end{array}$ & $\begin{array}{l}4(1.5) \\
{[0.4-3.9]}\end{array}$ & $\begin{array}{l}1.7 \\
(0.6-5.4)\end{array}$ & 0.93 & $\begin{array}{l}10(2.9) \\
{[1.4-5.3]}\end{array}$ & $\begin{array}{l}6(1.6) \\
{[0.6-3.4]}\end{array}$ & $\begin{array}{l}1.9 \\
(0.7-5.2)\end{array}$ & 0.94 \\
\hline VRE & $\begin{array}{l}53(11.5) \\
{[8.8-14.8]}\end{array}$ & $\begin{array}{l}13(5.0) \\
{[2.7-8.3]}\end{array}$ & $\begin{array}{l}2.5 \\
(1.3-4.7)\end{array}$ & 0.04 & $\begin{array}{l}46(13.5) \\
{[10.0-17.6]}\end{array}$ & $\begin{array}{l}20(5.3) \\
{[3.2-8.0]}\end{array}$ & $\begin{array}{l}2.8 \\
(1.6-4.9)\end{array}$ & 0.002 \\
\hline CRE & $\begin{array}{l}22(4.8) \\
{[3.0-7.2]}\end{array}$ & $\begin{array}{l}6(2.3) \\
{[0.8-4.9]}\end{array}$ & $\begin{array}{l}2.1 \\
(0.9-5.4)\end{array}$ & 0.93 & $\begin{array}{l}20(5.9) \\
{[3.6-8.9]}\end{array}$ & $\begin{array}{l}8(2.1) \\
{[0.9-4.1]}\end{array}$ & $\begin{array}{l}2.9 \\
(1.3-6.7)\end{array}$ & 0.09 \\
\hline \multicolumn{9}{|l|}{ Organisms } \\
\hline Klebsiella spp. & $\begin{array}{l}216(47.1) \\
{[42.4-51.7]}\end{array}$ & $\begin{array}{l}79(30.2) \\
{[24.7-36.1]}\end{array}$ & $\begin{array}{l}2.1 \\
(1.5-2.8)\end{array}$ & $<0.001$ & $\begin{array}{l}182(53.4) \\
{[47.9-58.8]}\end{array}$ & $\begin{array}{l}113(29.7) \\
{[25.2-34.6]}\end{array}$ & $\begin{array}{l}2.7 \\
(2.0-3.7)\end{array}$ & $<0.001$ \\
\hline Escherichia coli & $\begin{array}{l}174(37.9) \\
{[33.5-42.5]}\end{array}$ & $\begin{array}{l}95(36.3) \\
{[30.4-42.4]}\end{array}$ & $\begin{array}{l}1.1 \\
(0.8-1.5)\end{array}$ & 0.66 & $\begin{array}{l}141(41.3) \\
{[36.1-46.8]}\end{array}$ & $\begin{array}{l}128(33.7) \\
{[28.9-38.7]}\end{array}$ & $\begin{array}{l}1.4 \\
(1.0-1.9)\end{array}$ & 0.17 \\
\hline Citrobacter spp. & $\begin{array}{l}31(6.8) \\
{[4.6-9.4]}\end{array}$ & $\begin{array}{l}14(5.3) \\
{[3.0-8.8]}\end{array}$ & $\begin{array}{l}1.3 \\
(0.7-2.5)\end{array}$ & 0.66 & $\begin{array}{l}23(6.7) \\
{[4.3-9.9]}\end{array}$ & $\begin{array}{l}22(5.8) \\
{[3.7-8.6]}\end{array}$ & $\begin{array}{l}1.2 \\
(0.6-2.2)\end{array}$ & 0.94 \\
\hline $\begin{array}{l}\text { Pseudomonas } \\
\text { aeruginosa }\end{array}$ & $\begin{array}{l}46(10.0) \\
{[7.4-13.1]}\end{array}$ & $\begin{array}{l}6(2.3) \\
{[0.8-4.9]}\end{array}$ & $\begin{array}{l}4.8 \\
(2.0- \\
11.3)\end{array}$ & 0.001 & $\begin{array}{l}42(12.3) \\
{[9.0-16.3]}\end{array}$ & $\begin{array}{l}10(2.6) \\
{[1.3-4.8]}\end{array}$ & $\begin{array}{l}5.2 \\
(2.6-10.5)\end{array}$ & $<0.001$ \\
\hline Enterobacter spp. & $\begin{array}{l}88(19.2) \\
{[15.7-23.1]}\end{array}$ & $\begin{array}{l}20(7.6) \\
{[4.7-11.5]}\end{array}$ & $\begin{array}{l}2.9 \\
(1.7-4.8)\end{array}$ & $<0.001$ & $\begin{array}{l}80(23.5) \\
{[19.1-28.3]}\end{array}$ & $\begin{array}{l}28(7.4) \\
{[5.0-10.5]}\end{array}$ & $\begin{array}{l}3.9 \\
(2.4-6.1)\end{array}$ & $<0.001$ \\
\hline Enterococcus spp. & $\begin{array}{l}354(77.1) \\
{[73.0-80.9]}\end{array}$ & $\begin{array}{l}94(35.9) \\
{[30.1-42.0]}\end{array}$ & $\begin{array}{l}6.0 \\
(4.3-8.4)\end{array}$ & $<0.001$ & $\begin{array}{l}296(86.8) \\
{[82.7-90.2]}\end{array}$ & $\begin{array}{l}152(40.0) \\
{[35.0-45.1]}\end{array}$ & $\begin{array}{l}9.9 \\
(6.8-14.4)\end{array}$ & $<0.001$ \\
\hline Aeromonas spp. & $\begin{array}{l}10(2.2) \\
{[1.0-4.0]}\end{array}$ & $\begin{array}{l}3(1.1) \\
{[0.2-3.3]}\end{array}$ & $\begin{array}{l}1.9 \\
(0.5-7.0)\end{array}$ & 0.66 & $\begin{array}{l}10(2.9) \\
{[1.4-5.3]}\end{array}$ & $\begin{array}{l}3(0.8) \\
{[0.2-2.3]}\end{array}$ & $\begin{array}{l}3.8 \\
(1.0-13.9)\end{array}$ & 0.17 \\
\hline $\begin{array}{l}\text { Other Gram-negative } \\
\text { rods, NOS }\end{array}$ & $\begin{array}{l}47(10.2) \\
{[7.6-13.4]}\end{array}$ & $\begin{array}{l}11(4.2) \\
{[2.1-7.4]}\end{array}$ & $\begin{array}{l}2.6 \\
(1.3-5.1)\end{array}$ & 0.03 & $\begin{array}{l}42(12.3) \\
{[9.0-16.3]}\end{array}$ & $\begin{array}{l}16(4.2) \\
{[2.4-6.7]}\end{array}$ & $\begin{array}{l}3.2 \\
(1.8-5.8)\end{array}$ & 0.001 \\
\hline Anaerobes & $\begin{array}{l}35(7.6) \\
{[5.4-10.4]}\end{array}$ & $\begin{array}{l}29(11.1) \\
{[7.5-15.5]}\end{array}$ & $\begin{array}{l}0.7 \\
(0.4-1.1)\end{array}$ & 0.66 & $\begin{array}{l}30(8.8) \\
{[6.0-12.3]}\end{array}$ & $\begin{array}{l}34(8.9) \\
{[6.3-12.3]}\end{array}$ & $\begin{array}{l}1.0 \\
(0.6-1.6)\end{array}$ & 0.94 \\
\hline
\end{tabular}

more common in the presence of a prior biliary ES: Enterococcus spp. $(77.1 \%$ [ $95 \% \mathrm{Cl} 73.0 \%-80.9 \%]$ vs. $35.9 \%$ [95\% $\mathrm{Cl} 30.1 \%-$ 42.0\%]; OR6.0); Enterobacter spp. (19.2\% [95\%Cl15.7\%-23.1\%] vs. $7.6 \%$ [95\%Cl4.7\%-11.5\%]; OR2.9); Pseudomonas aeruginosa $(10.0 \%$ [ $95 \% \mathrm{Cl} .4 \%-13.1 \%]$ vs. $2.3 \%$ [ $95 \% \mathrm{Cl} 0.8 \%-4.9 \%]$; OR4.8); and Klebsiella spp. (47.1\% [95\%Cl42.4\%-51.7\%] vs. $30.2 \%$ [95\% Cl24.7\%-36.1\%]; OR2.1).

Likewise, the presence of a biliary stent significantly increased the probability of VRE $(13.5 \%$ [95\%Cl10.0\%-17.6\%] vs. $5.3 \%$ [95\% Cl3.2\%-8.0\%]; OR2.8). A similar effect regarding the increased presence of certain organisms was seen in the presence of a prior biliary stent: Enterococcus spp. $86.8 \%(95 \%$ $\mathrm{Cl} 82.7 \%-90.2 \%)$ vs. $40.0 \%$ (95\%Cl35.0\%-45.1\%; OR9.9); Enterobacter spp. $23.5 \%$ (95\%Cl19.1\%-28.3\%) vs. $7.4 \%$ (95\% CI5.0\%-10.5\%; OR3.9); P.aeruginosa $12.3 \%$ (95\%Cl9.0\%-16.3\%) vs. $2.6 \%$ (95\% Cl1.3\%-4.8\%; OR5.2); Klebsiella spp. 53.4\% (95\% $\mathrm{Cl} 47.9 \%-58.8 \%)$ vs. $29.7 \%(95 \% \mathrm{Cl} 25.2 \%-34.6 \%$; OR2.7). There was no effect on the incidence of E.coli with regard to the presence of a biliary stent or anaerobes with regard to the presence of biliary ES or a biliary stent. 


\section{Discussion}

This single-center retrospective study evaluated the microbiology of biliary aspirates obtained from 721 ERCP procedures in patients with suspected acute cholangitis. Positive bile cultures were found in nearly $92 \%$ of these ERCPs. In general, duodenoscope cleaning techniques, culture appropriation techniques, and processing have remained predominantly consistent, with only minor variations [17] from the time of a prior study on this topic published by our institution in 2002 [12].

This study covered a 7-year time period between 2010 and 2016, similar to the previously published bile culture data from our institution for a comparable group of patients covering a 7-year time period between 1994 and 2000. The current study however contains over four times more ERCPs with bile aspiration performed than in the prior study (721 ERCP procedures compared with 180 ERCP procedures), owing to near universal adoption of the practice by all gastroenterologists performing ERCP for this indication at our institution.

The current study confirms and expands upon the prior data that there is an increased incidence in polymicrobial bile cultures, with an increased likelihood of Enterococcus spp. in the setting of an indwelling biliary stent [12]. We also found a significantly higher likelihood of Enterobacter spp. (23.5\% vs. $7.4 \%)$, P.aeruginosa (12.3\% vs. $2.6 \%$ ), and K.pneumoniae $(53.4 \%$ vs. $29.7 \%)$ in the presence of a biliary stent, which was not appreciated in the prior study. We suspect this association was uncovered in this study because of the much larger sample size and expanded culturing of bile in nearly all patients suspected of having acute cholangitis.

The current study also realized a higher positive bile culture rate in the patients who had not had a prior biliary stent. We found that bile cultures were positive in $86.1 \%$ of patients who had previously not had a biliary stent, whereas only $55 \%$ of patients in the prior study had a positive bile culture without a prior biliary stent. We suspect this may be related to more patients undergoing ERCP with biliary aspiration who have possibly had other sources of their clinical symptoms, longer duration of antibiotics pre-ERCP, or different antibiotics pre-ERCP, or to there being less resistance to antibiotics in biliary organisms in the prior study.

In the current study, we sought to clarify the influence of a prior biliary ES on the positivity rate and microbiology of bile cultures. We found effects of biliary ES that were similar to that of having a prior indwelling biliary stent. This is to be expected as both biliary ES and an indwelling biliary stent violate the natural sphincter of Oddi barrier between the duodenal lumen and the biliary system [20]. We hypothesize the changes in microbiology with regard to the presence or absence of a biliary ES or a biliary stent is likely due to migration of luminal bacteria into the biliary tree and/or colonization of the existing biliary stent. This is further supported by the increased presence, in these subsets of patients, of organisms that are well-known to produce biofilms and colonize foreign bodies such has urinary catheters and biliary stents (Enterobacter spp., Enterococcus spp., Klebsiella spp., and Pseudomonas spp.) [21-24]. There is a large overlap of patients with a biliary ES and an existing or prior biliary stent. In addition, although the data are not completely available to analyze within this study, patients who receive a biliary ES or biliary stent likely have a higher disease burden from more complex disease, prior hospitalizations, and multiple exposures to antibiotics, which may contribute to higher rates of positive bile culture, antibiotic resistance, and bacterial colonization.

We discovered a notable number of high concern multidrug-resistant organisms (155/662; 23.4\%). There was a significant increase in isolation of VRE in the presence of a biliary ES (11.5\% vs. 5\%; OR2.5 [95\%Cl1.3-4.7]; adjusted $P=0.04$ ] and biliary stents [13.5\% vs. 5.3\%; OR2.8 [95\%Cl1.6-4.9]; adjusted $P=0.002$ ], but not other multidrug-resistant organisms. This association has been reported previously [25].

A potential benefit of collecting and culturing bile from patients with suspected cholangitis is the ability to tailor antibiotic regimens to the resistance pattern of the biliary aspirates. Furthermore, in patients with recurrent cholangitis from structural disease (e.g. primary sclerosing cholangitis), the presence of recurrent positive biliary cultures or highly resistant biliary cultures may prompt liver transplant teams to advocate for exception points for liver transplantation. We also believe that, in the era of known risk of transmission of organisms from reusable duodenoscopes, the knowledge of biliary culture data can inform the decision regarding enhanced endoscope reprocessing and/or future endoscope usage for that patient. In a patient with a known multidrug-resistant organism on bile culture, we would consider reprocessing that endoscope subsequently with an enhanced treatment (ethylene oxide sterilization). Furthermore, we would consider future ERCPs for that patient being done with a single-use duodenoscope.

The recommended choice of empiric antibiotics by medical societies for community acquired biliary infections include a third-generation cephalosporin, a fluoroquinolone, or a penicillin/beta-lactamase inhibitor $[2,13,26]$. In our earlier study, given the $96 \%$ susceptibility of Gram-negative organisms to ciprofloxacin, we continued to consider ciprofloxacin a recommended initial empiric choice for suspected acute cholangitis. In the current study however the susceptibility to ciprofloxacin of the most common Gram-negative organisms has been reduced substantially (E.coli susceptibility $64 \%$ and K.pneumoniae susceptibility $88 \%$ ). This decrease in susceptibility to ciprofloxacin demonstrates an increase in the prevalence of Gram-negative organisms resistant to this commonly used antibiotic in our community. A similar change in resistance pattern has been reported in other geographic areas as well [27-29]. An important strength of this study is that we were able to assess a longitudinal change over time in the antibiotic sensitivity of biliary organisms by comparing with a similar cohort from our prior study [12].

Antibiotic selection for suspected cholangitis is an important decision made routinely in clinical practice. We support a judicious use of empiric antimicrobial agents for biliary infections that should be modified based on the local antibiogram. We no longer use ciprofloxacin as an empiric antibiotic for suspected acute cholangitis. For inpatients suspected of having acute cholangitis, the practice at our institution is to recom- 
mend the empiric use of intravenous fourth-generation cephalosporin (i.e. cefepime) and intravenous metronidazole, as the combination is active against the most commonly isolated biliary organisms and also against the SPICE organisms (Serratia spp., Pseudomonas spp., Indole-positive Proteus spp., Citrobacter spp., Enterobacter spp., Providentia spp., Morganella spp., and Acinetobacter spp.), which may have inducible resistance (amp-C gene) to intravenous extended-spectrum beta-lactam antibiotics, such as piperacillin-tazobactam and ceftriaxone. After ERCP with effective biliary drainage, a decision is made to de-escalate antibiotics to narrower spectrum oral options, pending evaluation of clinical improvement and preliminary bile culture and blood culture data. These preliminary culture data are available in 2-3days and finalized within 5 days. As a biliary infection with isolated Enterococcus spp. is unusual in the presence of an intact sphincter of Oddi barrier between the duodenal lumen and the biliary system, and polymicrobial infections with Enterococcus spp. do not require enterococcal coverage [26], we do not recommend routine empiric or directed antibiotic coverage for this organism.

The limitations of this study include that it is a single-center retrospective study conducted at a tertiary academic healthcare center. Given the retrospective nature of the study, it is possible that a small percentage of patients undergoing ERCP for suspected cholangitis did not have bile aspirated at the time of ERCP, which may introduce selection bias. Although we do not have data on the frequency of this occurring, we believe it is quite rare, given our clinical practice patterns. As microbial frequency and antimicrobial resistance patterns do have some geographic and regional variation, this may limit the generalizability of this study. Because all of the patients studied were at a tertiary academic healthcare center, the findings may not be applicable to community healthcare centers. However, as our patient population, and procedural and processing techniques have remained similar since a prior study performed on this topic at our institution, a historical control is available for this study, which allows a comparison of data over time.

As most patients are transferred from referring hospitals, we could not quantify all prior antibiotic exposure in this study or assess whether antibiotics were given prior to collection of blood or bile cultures. Antibiotic exposure during hospitalization prior to ERCP likely impacts the positivity of both bile and blood cultures, as well as the microbiology profile. For instance, bile cultures were negative in 11 of the 307 patients (3.6\%) with pus visualized. This could be due to patients receiving antibiotics prior to the ERCP, reducing the positivity of the cultures. Another factor could be a descriptive error by the endoscopist in describing non-purulent dark or murky bile as pus.

In summary, this study demonstrates the susceptibilities of E.coli and Klebsiella spp. isolates to ciprofloxacin are significantly lower than historically noted. There was a substantial presence of serious antimicrobial-resistant organisms (ESBL-producing Enterobacteriaceae, MRSA, VRE, and CRE) identified in this study. The presence of an endobiliary stent or biliary ES is associated with differences in the microbiological characteristics and antimicrobial resistance patterns of the isolates. This is the only study to our knowledge where comparable cohorts of patients have been studied over time to demonstrate the changes in microbial frequency and changes to antimicrobial resistance patterns.

To develop an antibiogram, promote prompt titration of antimicrobial therapy, and decrease the risk of development of antimicrobial resistance, we advocate a biliary aspirate at the time of endobiliary interventions for suspected acute cholangitis and suggest it may be a marker for quality improvement at endoscopy centers worldwide. Furthermore, with recent appreciation of the risk of transmission of drug-resistant organisms via reusable duodenoscopes, we have used bile culture data to play a part in our algorithm for the reprocessing of reusable scopes and the selection of single-use scopes in selected patients.

\section{Acknowledgments}

We appreciate the input by Lois Bucksot RN, Lisa Terry RN, and Marnie Sieber RN who reviewed portions of this manuscript. This work was previously presented as Microbiology of ERCP bile aspirates in patients with suspected cholangitis at Digestive Disease Week 2018, Washington DC, USA, on 2 June 2018.

\section{Competing interests}

M.A. Gromski serves as a consultant for Boston Scientific. S. Sherman serves as a consultant for Boston Scientific, Cook Medical, and Olympus. G. Lehman serves as a consultant for Cook Endoscopy. J. Easler serves as a consultant for Boston Scientific. The remaining authors declare that they have no conflict of interest.

\section{References}

[1] Saik RP, Greenburg AG, Farris JM et al. Spectrum of cholangitis. Am J Surg 1975; 130: 143-150

[2] Miura F, Okamoto K, Takada T et al. Tokyo Guidelines 2018: initial management of acute biliary infection and flowchart for acute cholangitis. J Hepatobiliary Pancreat Sci 2018; 25: 31-40

[3] Rhodes A, Evans LE, Alhazzani W et al. Surviving sepsis campaign: International guidelines for management of sepsis and septic shock: 2016. Crit Care Med 2017; 45: 486-552

[4] van den Hazel SJ, Speelman P, Tytgat GN et al. Role of antibiotics in the treatment and prevention of acute and recurrent cholangitis. Clin Infect Dis 1994; 19: 279-286

[5] Lai EC, Mok FP, Tan ES et al. Endoscopic biliary drainage for severe acute cholangitis. NEJM 1992; 326: 1582-1586

[6] Park CS, Jeong HS, Kim KB et al. Urgent ERCP for acute cholangitis reduces mortality and hospital stay in elderly and very elderly patients. Hepatobiliary Pancreat Dis Int 2016; 15: 619-625

[7] Khashab MA, Tariq A, Tariq U et al. Delayed and unsuccessful endoscopic retrograde cholangiopancreatography are associated with worse outcomes in patients with acute cholangitis. Clin Gastroenterol Hepatol 2012; 10: 1157-1161

[8] Leese T, Neoptolemos JP, Baker AR et al. Management of acute cholangitis and the impact of endoscopic sphincterotomy. Br J Surg 1986; 73: 988-992

[9] Sugiyama M, Atomi Y. Treatment of acute cholangitis due to choledocholithiasis in elderly and younger patients. Arch Surg 1997; 132: $1129-1133$ 
[10] Tan M, Schaffalitzky de Muckadell OB, Laursen SB. Association between early ERCP and mortality in patients with acute cholangitis. Gastrointest Endosc 2018; 87: 185-192

[11] Xu MM, Carr-Locke DL. Early ERCP for severe cholangitis? Of course! Gastrointest Endosc 2018; 87: 193-195

[12] Rerknimitr R, Fogel EL, Kalayci C et al. Microbiology of bile in patients with cholangitis or cholestasis with and without plastic biliary endoprosthesis. Gastrointest Endosc 2002; 56: 885-889

[13] Mazuski JE, Tessier JM, May AK et al. The Surgical Infection Society revised guidelines on the management of intra-abdominal infection. Surg Infect 2017; 18: 1-76

[14] Yahav D, Franceschini E, Koppel F et al. Seven versus fourteen days of antibiotic therapy for uncomplicated gram-negative bacteremia: a non-inferiority randomized controlled trial. Clin Infect Dis 2019; 69: 1091-1098

[15] Perez KK, Olsen RJ, Musick WL et al. Integrating rapid diagnostics and antimicrobial stewardship improves outcomes in patients with antibiotic-resistant Gram-negative bacteremia. J Infect 2014; 69: 216225

[16] Uno S, Hase R, Kobayashi M et al. Short-course antimicrobial treatment for acute cholangitis with Gram-negative bacillary bacteremia. Int J Infect Dis 2017; 55: 81-85

[17] Rex DK, Sieber M, Lehman GA et al. A double-reprocessing high-level disinfection protocol does not eliminate positive cultures from the elevators of duodenoscopes. Endoscopy 2018; 50: 588-596

[18] US Food and Drug Administration. Supplemental measures to enhance duodenoscope reprocessing: FDA Safety Communication. 2017: Available from (Accessed 30.03.2022): http://wayback.archive-it.org/7993/20170722150658/https:/www.fda.gov/MedicalDevices/Safety/AlertsandNotices/ucm454766.htm

[19] US Food and Drug Administration. Olympus validates new reprocessing instructions for model TJF-Q180V duodenoscopes. FDA Safety Communication; 2017: Available from (Accessed 30.03.2022): http://wayback.archive-it.org/7993/20170722213115/https://www. fda.gov/MedicalDevices/Safety/AlertsandNotices/ucm439999.htm

[20] Kozarek R, Hovde O, Attia F et al. Do pancreatic duct stents cause or prevent pancreatic sepsis? Gastrointest Endosc 2003; 58: 505-509

[21] Hashem YA, Amin HM, Essam TM et al. Biofilm formation in enterococci: genotype-phenotype correlations and inhibition by vancomycin. Sci Rep 2017; 7: 5733

[22] Lee K, Yoon SS. Pseudomonas aeruginosa biofilm, a programmed bacterial life for fitness. J Microbiol Biotechnol 2017; 27: 1053-1064

[23] Paterson DL. Resistance in gram-negative bacteria: enterobacteriaceae. Am J Med 2006; 119: S20-S28 discussion S62-S70

[24] Piperaki ET, Syrogiannopoulos GA, Tzouvelekis LS et al. Klebsiella pneumoniae: virulence, biofilm and antimicrobial resistance. Pediatr Infect Dis J 2017; 36: 1002-1005

[25] Reuken PA, Torres D, Baier M et al. Correction: Risk factors for multidrug resistant pathogens and failure of empiric first-line therapy in acute cholangitis. PloS One 2017; 12: e0172373

[26] Solomkin JS, Mazuski JE, Bradley JS et al. Diagnosis and management of complicated intra-abdominal infection in adults and children: guidelines by the Surgical Infection Society and the Infectious Diseases Society of America. Clin Infect Dis 2010; 50: 133-164

[27] Chandra S, Klair JS, Soota K et al. Endoscopic retrograde cholangiopancreatography-obtained bile culture can guide antibiotic therapy in acute cholangitis. Dig Dis 2019; 37: 155-160

[28] Kaya M, Beştaş R, Bacalan F et al. Microbial profile and antibiotic sensitivity pattern in bile cultures from endoscopic retrograde cholangiography patients. World J Gastroenterol 2012; 18: 3585-3589

[29] Voiosu TA, Bengus A, Haidar A et al. Antibiotic prophylaxis prior to elective ERCP does not alter cholangitis rates or shorten hospital stay: results of an observational prospective study of 138 consecutive ERCPS. Maedica 2014; 9: 328-332 